\title{
AC 2011-2672: ARM/FPGA/I2C SENSOR NETWORK DEVELOPMENT AND TEACHING PLATFORM
}

\author{
Antonio Francisco Mondragon, Rochester Institute of Technology
}

Antonio F. Mondragon-Torres received the B.Sc. degree with honors from Universidad Iberoamericana, Mexico City, Mexico, the M.Sc. degree from Universidad Nacional Autnoma de Mexico, Mexico City, Mexico, and the Ph.D. degree (as a Fullbright-CONACYT scholarship recipient) from Texas A\&M University, College Station; all degrees in Electrical Engineering in 1990, 1996, and 2002, respectively. From 1988 to 1995, he worked in a telecommunications company TVSCOM, Mexico City, Mexico, designing teletext products, first as a Design Engineer and later as a Design Manager. In 1995, he joined the Mechanical and Electrical Department, Universidad Iberoamericana as an Associate Professor. From 2002 through 2008 he was with the DSPS R\&D Center's Mobile Wireless Communications Technology branch, Texas Instruments Dallas, TX and in 2008 he moved to the nanoMeter Analog Integration Wireless branch where he worked as Analog IP verification technical lead. In 2009 he worked for Intel Guadalajara, Design Center in Mexico as Front-End/Back-End technical lead. In 2009 he joined the Electrical, Computer and Telecommunications Engineering Technology Department at the Rochester Institute of technology where he currently is a tenured track assistant professor. His research interests are analog and digital integrated circuit implementation of communications systems, and System-on-a-Chip methodologies.

\section{Prafull Purohit, Rochester Institute of Technology}

Prafull Purohit received a B.E. degree in electronics engineering at Nagpur University, India in 2007. After completing his B.E., he joined the faculty in the Department of Electronics Engineering at Nagpur University, India. He is currently working towards M.S. in Electrical Engineering at Rochester Institute of Technology. His research activities include digital logic design, system-on-a-programmable-chip, and micro-controller based systems. 


\title{
ARM/FPGA/I2C Sensor Network Development and Teaching Platform
}

\begin{abstract}
In past few years we have seen many courses offered separately for embedded systems based on various industry standard 8/16/32-bit micro-controllers; their programming languages like $\mathrm{C} / \mathrm{C}++$ /assembly language; operating systems concepts and for digital system design based on FPGAs and CPLDs. Some courses were also focused on wireless networking standards like Bluetooth, Wi-Fi and ZigBee; interfacing different peripherals like liquid crystal displays (LCD), analog to digital and digital to analog conversion (ADC/DAC), synchronous serial communications based devices, memories, LED controllers, switches, etc. There is a strong need for a platform to include some of the key features of these different courses to teach with a unified development environment which will give student an opportunity to learn product development in an industry-like environment. Therefore we are introducing here a new development and teaching platform around an ARM/FPGA/I2C concepts which includes key features like industry standard 32-bit ARM processor, high density Altera FPGA, debug ports for external logic analyzers, access to communication protocols like UART, SPI, I2C to interface with external components, and the ability to create wired and wireless sensor networks.
\end{abstract}

\section{Introduction}

The computer engineering technology program has a very well-built course sequence in digital and embedded systems design with a strong emphasis on hands-on experience for the students. The last module in the sequence is being taught as a capstone course that is geared more towards industrial like applications and industrial design environment. This includes project management techniques, version control and project sign-off milestones.

Based on the above guidelines, we are developing a design experience for the students based on a popular commercial computer architecture such as the ARM processor[1, 2]; the flexibility of extending this architecture by creating hardware accelerator blocks by the integration of a high density FPGA; and by the addition of off-the-shelf sensor components that are interconnected through the popular I2C communications bus.

During the course, the students will be assigned the following tasks: assemble and test their PCB boards, which are mostly surface mount devices; bring up power management devices on board; familiarize with the ARM architecture; analyze and develop the optimal hardware and software partitions to allow system's maintenance and future upgradeability; identification of already available intellectual property blocks for reutilization; use of external logic analyzer; extend the ARM architecture by designing hardware accelerators; use of internal FPGA debugging capabilities; analyze system's power consumption and use of advanced power optimization techniques; explore pipelined architectures and their impact on algorithm's speedup; attain timing closure given system's specifications; work with a tight area/resources budget while maintaining system's performance; create a sensors communication network by using on board I2C devices and possibly a wireless network by using ZigBee or other similar standard.

Out of this capstone experience the students must be comfortable to work in an industry type environment by: working in teams, selecting leadership, work towards achievable milestones, work on a version control environment, work under a bug tracking system, work on documentation deliverables, demonstrate intermediate and final project's prototyping phases. 
As mentioned this is the original purpose of the development and teaching platform, but as we progress through the paper we will expose that the platform could be suited as an aid in some other courses.

\section{Objectives}

The main objective behind such a platform is to demonstrate some practical examples of product development and application design by combining different aspects of electronics and digital hardware design, embedded systems design, wired and wireless communication protocols usage and printed circuit board (PCB) design[3].

Another practical aspect of this platform is that most of the components have been donated by different companies (e.g. ARM, Altera, Texas Instruments) and this is the result of merging the solutions from different vendor under a single platform that will provide students with a wide visibility into what the types of design they will be exposed to whenever they join the workforce.

\section{Platform components}

This platform includes both hardware and software components required for designing a highly sophisticated embedded system. Hardware components include a commercial Evauator-7T ARM-7TDMI development board, the complete design including switching power supplies for a FPGA based daughter-card with I2C networking capabilities and for students to design different sensor modules accessible through the I2C network. In addition, built in interfaces for SPI as well as USB are incorporated. On other hand, software components required to develop in this platform include ARM developer suite (ADS), Quartus II and Modelsim.

\section{Evaluator-7T board}

The Evaluator-7T board is a simple ARM platform that includes a minimal set of core facilities. Figure 1 shows a basic block diagram of Evaluator-7T development board and Figure 2 shows a photograph of the actual Evaluator-7T board. It is powerful and flexible enough to function as an introductory evaluation platform for ARM technology. The board enables you to:

- Download and debug programs.

- Attach additional input/output and peripherals for experimentation.

The Evaluator-7T board contains the following major components:

- Samsung KS32C50100 microcontroller (ARM-7TDMI)

- $512 \mathrm{~KB}$ flash EPROM

- 512KB SRAM

- two 9-pin D-type RS232 connectors

- reset and interrupt push buttons

- four user-programmable LEDs and a 7-segment LED display

- 4-way user input DIP switch

- $3.3 \mathrm{~V}$ voltage regulator

Students can start using this board as early as second year on a basic Microcontroller course learning how to program in assembly and $C$ language, as well as to visualize the operations by using and external logic analyzer. These boards were donated by ARM to support and encourage the learning of this important embedded systems architecture. 


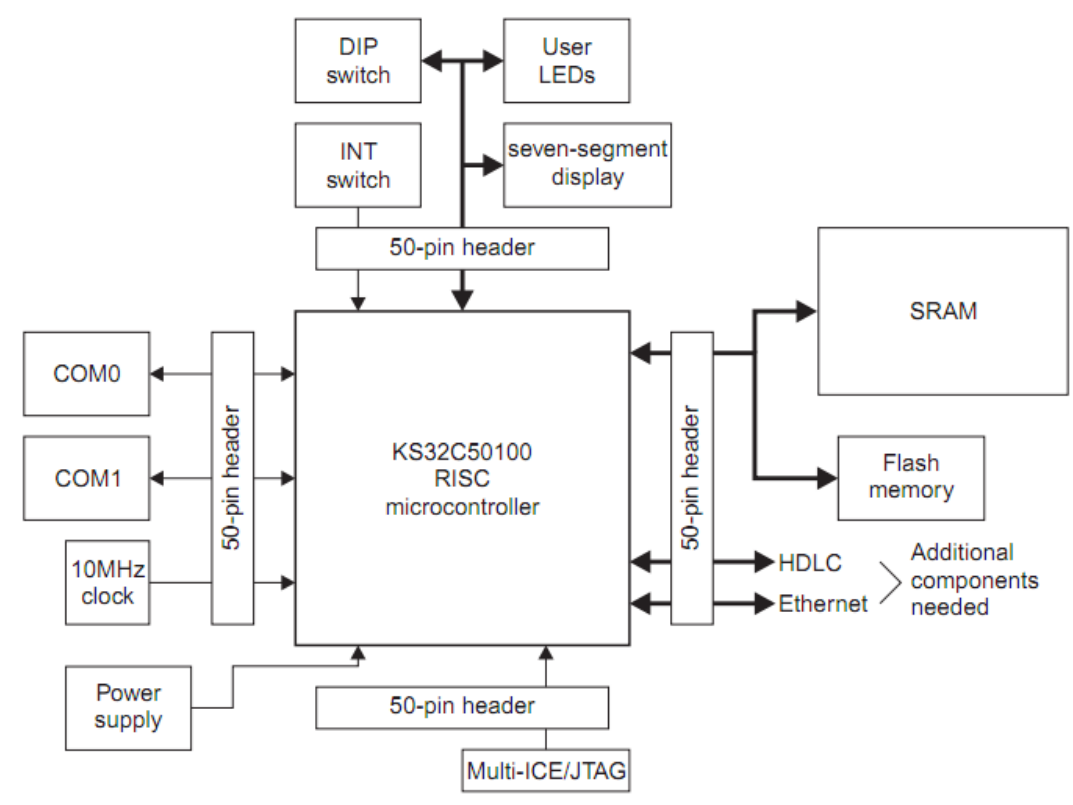

Figure 1 - Evaluator-7T block diagram

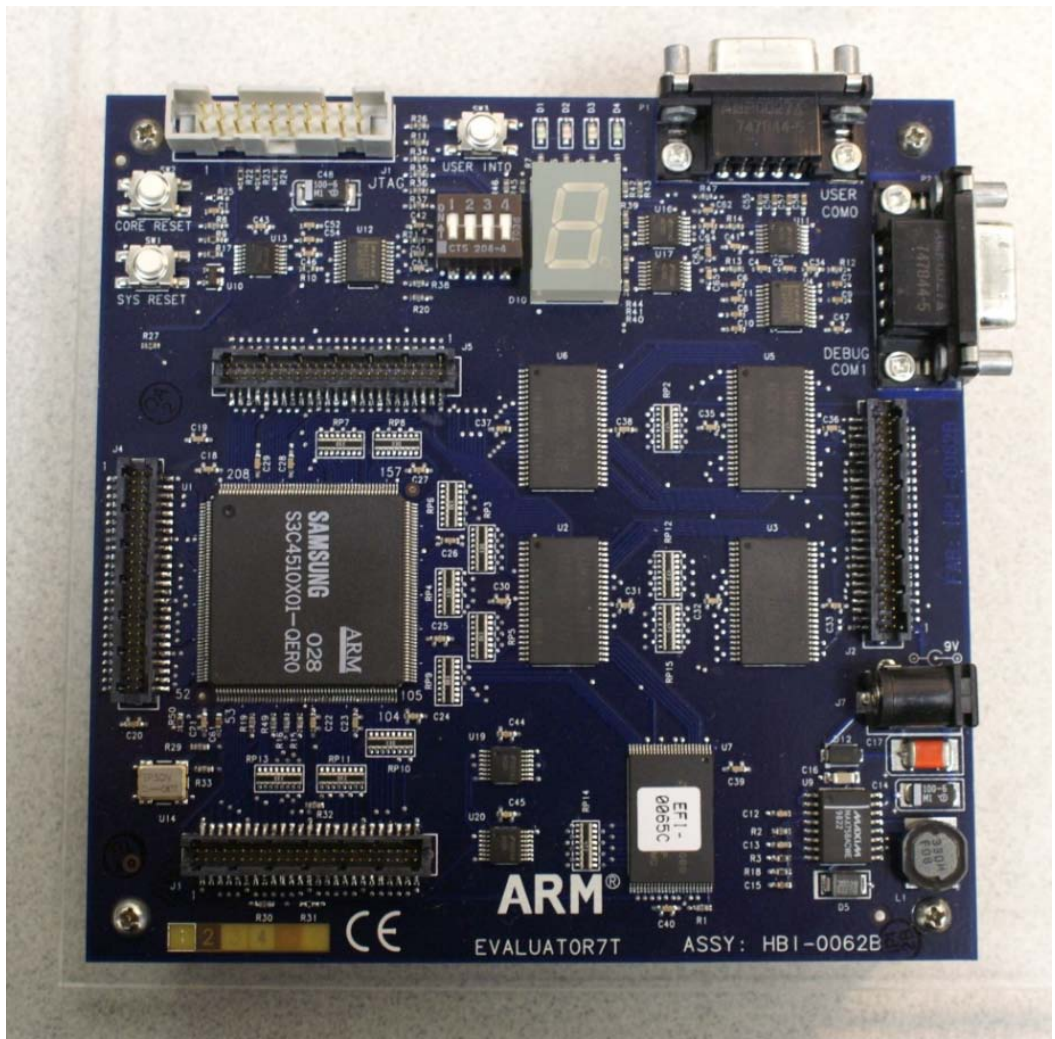

Figure 2 ARM7 Development Board (Evaluator-7T)

\section{FPGA daughter-card}

The FPGA daughter-card was designed as an addition to the ARM Evaluator-7T board. It extends the capabilities of ARM so that it can be used in some high computing applications with special hardware required for particular application that are to be implemented on the FPGA. Some of these applications could be DSP related algorithms, hardware acceleration, coprocessors, multiprocessor collaboration, floating-point arithmetic, fast memory interface, multipliers, reconfigurable hardware, parallel processing, etc. The daughter-card is aimed for the 
student to assemble it and bring it up one stage at a time. The daughter card by itself could be used in introductory and advanced courses on HDL as well as on advanced courses on microcontrollers. The complete system could be used on embedded systems courses as well as to develop a senior project. Now the different subsystems available in the daughter card will be described in more detail.

\section{Switched mode power supplies (SMPS)}

The first step is to design the switched mode power supplies. Texas Instruments provides several solutions for the design of power supplies and it has a set of products aimed at FPGA circuits. One characteristic of modern electronic design is that there are several power supplies for different components. For example, most of the components in the daughter-card will work with $3.3 \mathrm{~V}$, since the Evaluator-7T provides this voltage, it can be routed directly to the daughter-card, but in the other hand if the amount of current that provide the Evaluator-7T is not enough, the board should be able to convert form for example $5 \mathrm{~V}$ to $3.3 \mathrm{~V}$. Also the daughter-card can operate independently from the ARM board. Now the FPGA requires $1.2 \mathrm{~V}$ for the core and 2.5 for PLLs and I/O, then two more supplies have to be derived from the common 3.3V. The student has to investigate which are the ranges of current required by the Cyclone III embedded in the daughter-card to design the corresponding power supplies. A programmable frequency generator requires $1.8 \mathrm{~V}$ for operation, this requires the student to design another power supply for this component, since it is not a high current device, a simple linear regulator such as an LDO could be used.

There are actually several very good references on semiconductor company sites that give students an overview on how power supplies for FPGAs are designed, their requirements and even free software to design them. e.g. www.ti.com/alterafpga.

\section{Logic analyzer probes}

Nowadays with the high level of integration in circuits, the development boards do not provide access to internal nodes such as data and control buses for the students. To explore and understand the basic mechanics on a microcontroller operation and how memory is accessed, all address-data-control buses are available for students to connect directly to a logic analyzer and understand the processed involved in microprocessor operations. This allows the students to use a hardware logic analyzer and understand fetch, decode and execute cycles as well as memory access. Our laboratories are equipped with a Tektronix TLA-714 logic analyzer per bench and thus the opportunity to use this board as a teaching and debugging platform.

\section{Field programmable gate array}

A field programmable gate array (FPGA) is included in the board for students to expand on the functions of the ARM core and also as a standalone hardware platform. The concept here is about interfacing among different hardware components. One application is for the ARM to see the FPGA as a coprocessor that will be customized for particular application. For example: digital filtering, matrix operations, data logging and averaging, etc. The Cyclone III integrated in the board is on a QFP package such that the student may be able to access some of the pins externally for debugging purposes, not very practical and safe, but the accessibility is better than on traditional BGA packages. The FPGAs were donated by Altera together with USB-Blaster JTAG debuggers and programmers that provide students with a very convenient way to program and debug the board. 


\section{Inter integrated circuit (I2C) network}

Since one of the objectives is to extend the use of this development system to enable students to explore different sensors for diverse applications, what we found as a common ground in many interfaces with sensors is that they can be classified in three ranges: Analog, I2C and SPI. The decision was made to provide an onboard I2C network in order to enable students to connect different sensors to the daughter-card efficiently. So if the sensor is analog, an I2C analog to digital component is added to a small sensor card to interface with the sensor. As mentioned many components already are I2C communications capable, and for those that require SPI communications an I2C to SPI bridge is included, so the student always sees the interface to any sensor as an I2C transaction. Most of the I2C components were available through Texas Instruments sample program and some of the sensors were acquired from other companies as samples. Modules are been designed in such a way that they can be tested by programming the ARM board or by the Cyclone III FPGA using an HDL language or a soft processor such as the Nios-II.

\section{Low speed wireless communications}

Since this platform is targeted for sensor network applications, an interface with popular radio frequency (RF) wireless motes was created. The standard for communication chosen was Zigbee which is very popular in short distance low speed wireless communications and it is one of the standards being used in smart grid developments for inside home/office communications among devices. Either the ARM or the FPGA can communicate with the Texas Instruments MSP-430 based wireless modules thus simplifying the interfacing. The RF sensor nodes were donated by Texas Instruments for this and other projects.

\section{Universal serial bus communications}

No modern embedded communications system lacks USB capabilities, for this purpose an asynchronous serial interface to USB is provided. The UART requires to be implemented on the FPGA, it can be generated as part of a complete system using an Altera NIOS-II processor and SOPC builder or it can be brought as a readymade component to be integrated with an HDL design. Unfortunately both UARTs on the Evaluator-7T board are already being used and the only way to use them is by modifying the board which is not recommended.

\section{Serial peripheral interface (SPI)}

SPI is another very popular serial interface that students will be able to explore, but in this particular case a bridge component is being used to translate from I2C to SPI, this simplifies the overall students experience and allows them to interface SPI sensors without the need to learn or code another communications standard.

\section{Other peripherals}

While the interfaces are limited, there is an accessible expansion connector that can be used for example to connect keyboards, LCD displays and other types of peripherals.

\section{Daughter-card design}

The complete design was done using Eagle PCB which is very popular among students, hobbyists and there is a very active community developing components and footprint libraries. This makes it easy for students to reuse and share libraries. The free version of the Eagle PCB has enough capabilities to design the small sensor cards. 


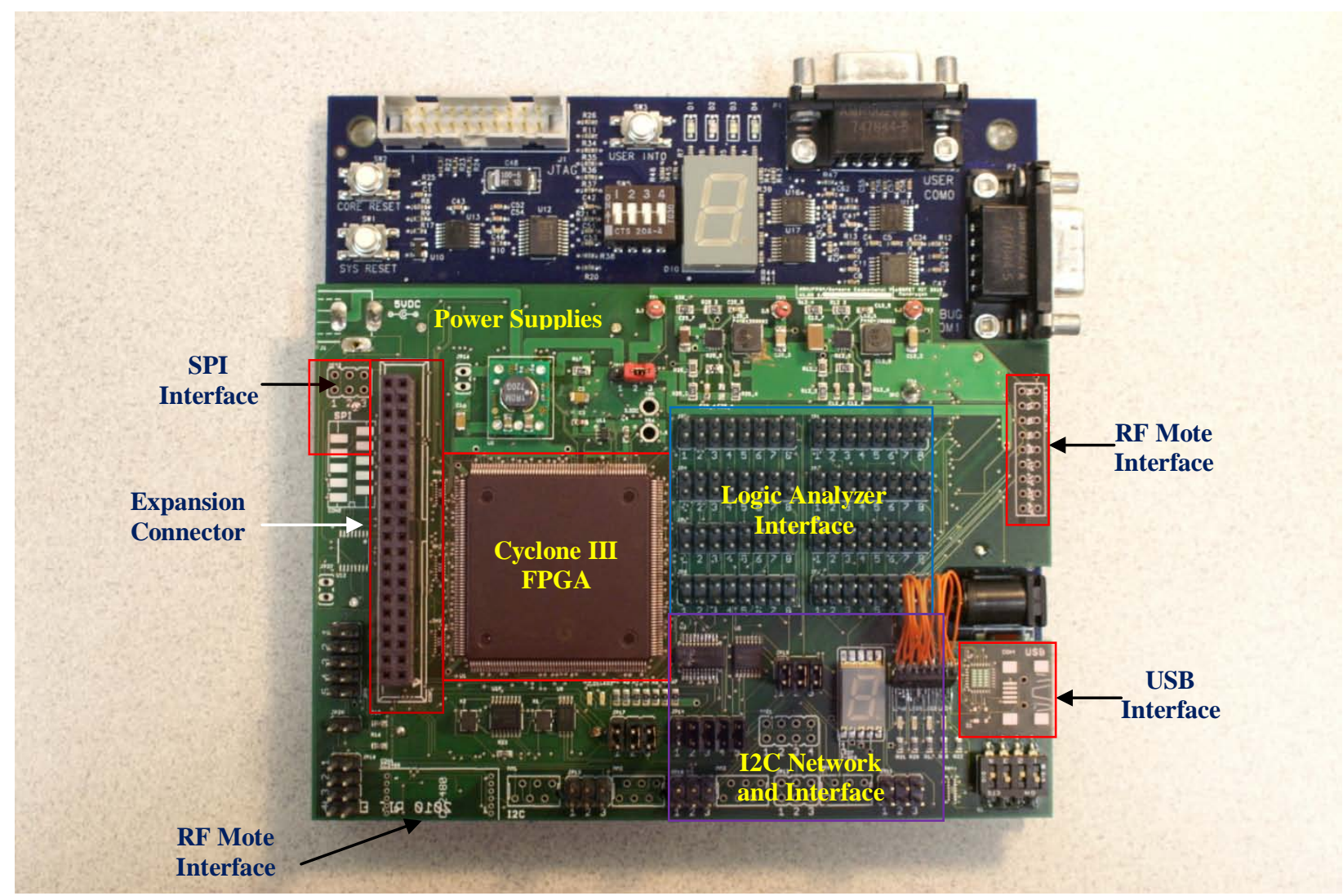

Figure 3 FPGA Daughter Card

\section{I2C based plug-and-play modules for sensor applications}

As shown in Figure 4, students are developing material and I2C based plug-and-play cards for laboratories and experiments based on the presented platform as part of their design experiments. The list of experiments, laboratories and design procedures that are being developed are:

- ARM/FPGA/I2C Development platform overview

- I2C communications protocol overview and use of protocol analyzer

- Digital I/O I2C Peripherals

- $\mathrm{A} / \mathrm{D}$ and $\mathrm{D} / \mathrm{A}$ converters with I2C control

- I2C controlled sensors (Temperature, Accelerometers, proximity, etc)

- $\quad$ I2C controlled non-volatile memory and potentiometers

- Digital Filter implementation on FPGAs

- Encryption algorithms implementation on FPGAs

As an example of the work being developed by these students, simple small boards were designed and manufactured and shown in Figure 4. Few of them were assembled and tested successfully. These boards contains I2C based parallel I/O expander, a 512KB EEPROM, digitally controlled potentiometer, 4x4 LED card, and 12-bit ADC \& DAC. This is an illustration on how students can start developing sensor cards for different purposes such as sensing: temperature, acceleration, humidity, pressure proximity, light intensity, color, etc. By using an on-site PCB milling machine the turnaround time to have it manufactured and assembled should not be more than couple days, thus a one week lab should allow the time for the student to design and test their sensor board. 


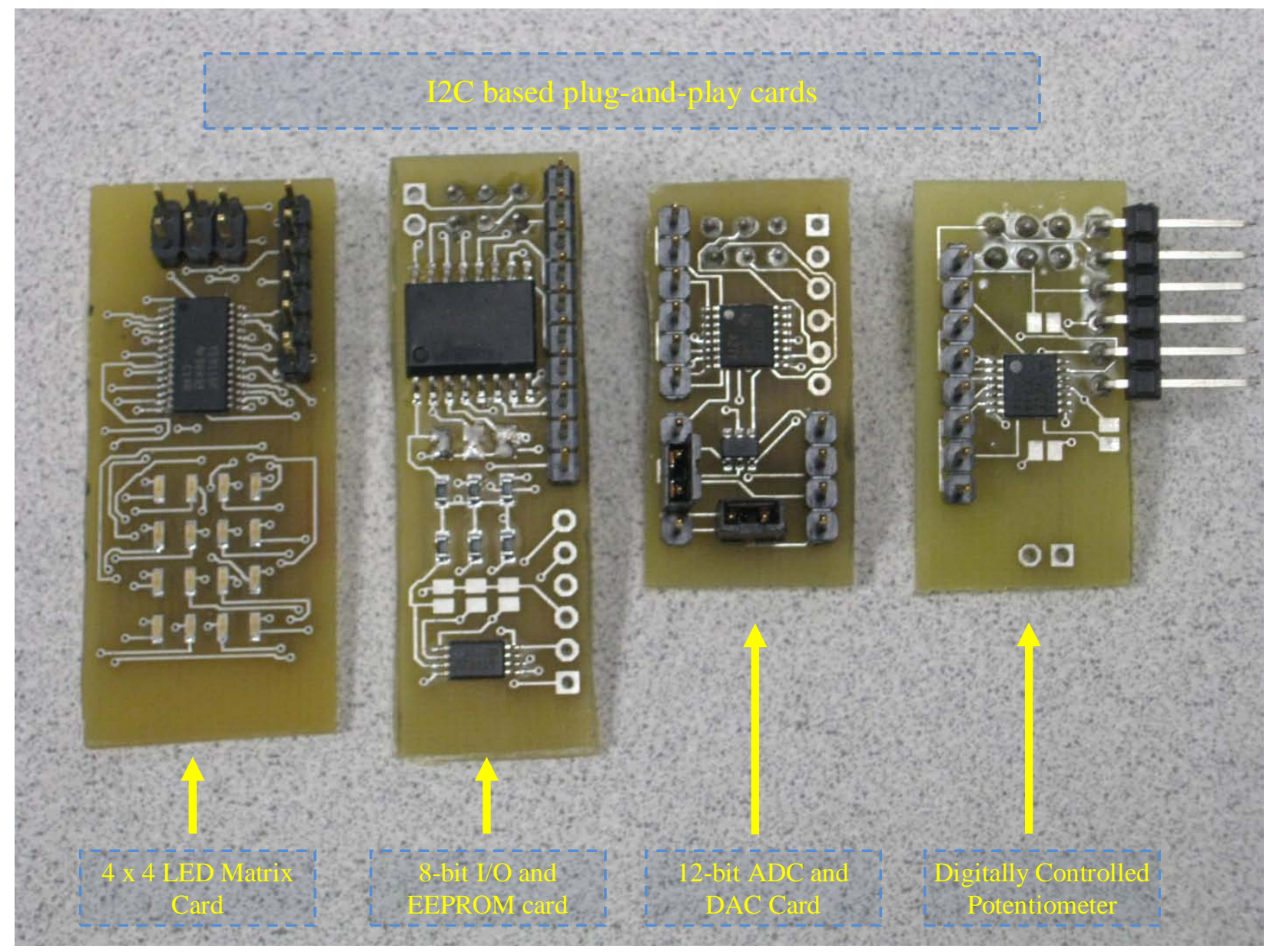

Figure 4. I2C based Card examples

\section{Electronic design automation (EDA) tools}

To develop with this board there is a set of EDA tools available for students in the laboratories, some of them require a license, but the majority of them are accessible to students at no cost. Here is a list of some of the programs that could be used to develop on this platform:

\begin{tabular}{|l|l|l|l|}
\hline \multicolumn{1}{|c|}{ EDA Tool } & \multicolumn{1}{c|}{ Vendor } & \multicolumn{1}{c|}{ Purpose } & \multicolumn{1}{c|}{ License Req. } \\
\hline ARM Developer Suite & ARM & Assembly \& C Code development & Yes/Academic \\
\hline GNU ARM & GNU & Assembly \& C/C++ Code development & No \\
\hline Quartus II/Nios II & Altera & FPGA Synthesis, Place \& Route & No/Limited \\
\hline Modelsim-Altera & Altera/MGC & Simulation & No/Limited \\
\hline Active HDL Student Ed & Aldec & Simulation & No/Limited \\
\hline Eagle PCB & Cadsoft & Schematic entry and PCB design & No/Limited \\
\hline
\end{tabular}




\section{Platform design overview}

This platform covers some of the attractive technologies or methodologies related to electronic and digital design as well as embedded systems, and sensor networks. Figure 5 shows a basic architectural block diagram of the complete ARM/FPGA/I2C teaching and development platform.

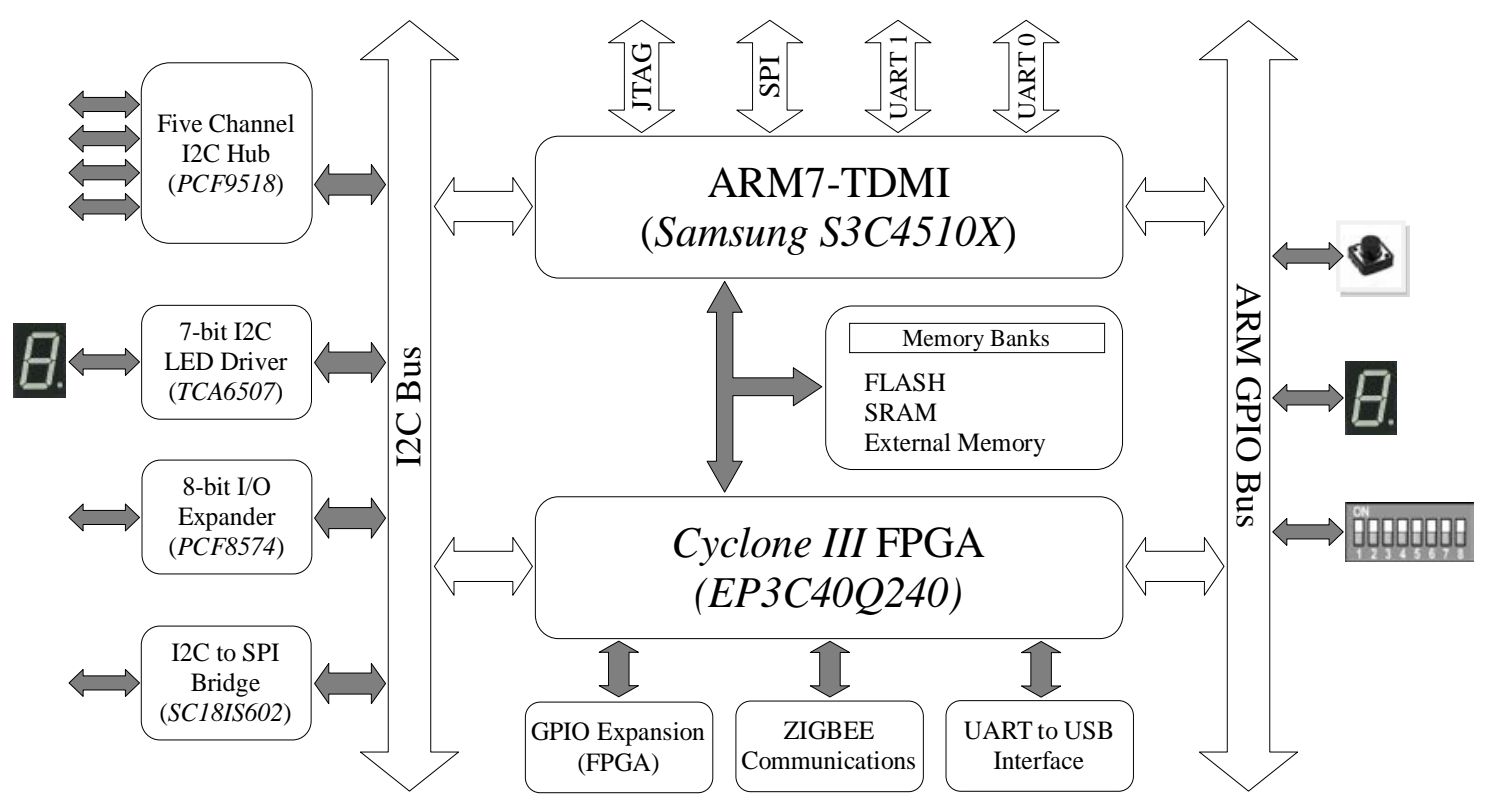

Figure 5. Block diagram of ARM-FPGA-Sensor based platform

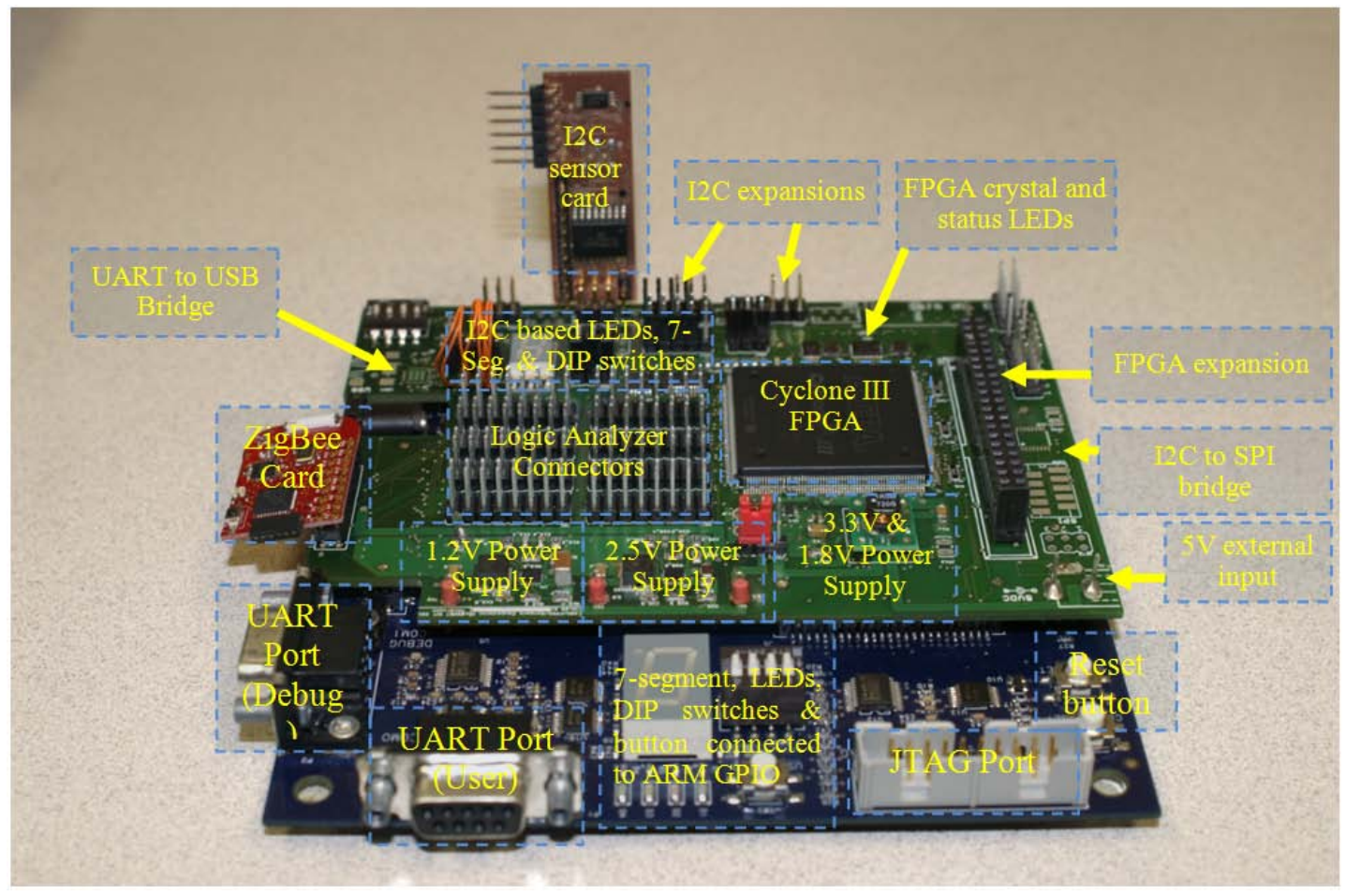

Figure 6 ARM/FPGA/I2C Sensor Platform

This platform can be used to design a separate course sequence in electronic system design or integrated in the current sequence at diverse levels. 


\section{Hardware design}

ARM-FPGA-Sensor platform can be used to teach different steps involved in product development from system specification to final product. A possible flow for hardware design based on this platform is given below:

- Defining system specification

- Schematic design

- $\quad$ Printed circuit board (PCB) design

- Hardware assembly

- Testing and debugging

\section{Digital design}

ARM-FPGA-Sensor platform explores the features of FPGA as a base for implementing different digital designs with a great flexibility. The FPGA daughter-card can be used separately to design and implement digital circuits, teach hardware description languages. Other possible use of this daughter-card could be in teaching other technologies like interfacing I2C based peripherals, hardware reconfiguration, co-processing by designing dedicated processing blocks in FPGA. Some of the common industrial applications are:

- LCD interface unit

- Keyboard interface unit

- Digital filters

- Encryption/Decryption units

- Nios-II processor

\section{Software design/ embedded systems}

An embedded system in simple words is nothing but an electronic system with some hardware/software components which is designed to execute particular tasks in the form of a program executing on the hardware components. The Evaluator-7T board can be used separately to showcase different aspects of software design in embedded system. This platform can be used to provide good understanding of micro-computer systems. Some of the possible applications of this platform in embedded system/ software design are:

- Introduction to assembler, linker, compiler

- Introduction to assembly, $\mathrm{C} / \mathrm{C}++$ languages

- Introduction to microcomputer design

- Memory interfacing

- Device drivers

\section{Communication and networking}

A good electronic system includes different communication and networking support to communicate with other systems and transfer data to each other. The ARM/FPGA/I2C sensor platform includes various communication and networking standards to communicate with other devices, interface different sensors, and wireless networking between different modules. It includes:

- $\quad$ I2C - Inter Integrated Circuits communication protocol

- $S P I$ - Serial to Peripheral Interface communication protocol

- USB - Universal Serial Bus

- UART - Universal Asynchronous Receiver/Transmitter

- ZigBee-RF - Low power wireless mesh networking standard 


\section{Hardware - software co-design}

There is always a tradeoff on what goes in software and what goes into hardware. The students must develop the criteria from system specifications to measure what the capabilities of the microcontroller are and implement those function that will be best suited for hardware implementation as a co-processor or hardware accelerator. Another aspect is to go over the level of coupling required being loose or tight. This platform could be thought as composed of a fixed part and a programmable part, being the programmable both hardware and software.

\section{Outcomes/advantages}

What are the benefits to students from using this platform? What will they get to learn? This section will contain some skills the students may be able to develop:

- Debugging and troubleshooting using a logic analyzer.

- Design on switched mode and linear based regulated DC power supply design

- Memory interfacing between a microprocessor/microcontroller and co-processors.

- Hardware/Software partitioning and co-design.

- Simple local networking concepts and signal processing.

- Basics of system design and product development by going through many of the phases required designing a product using this platform.

- As students work with this platform they will be encouraged to document and make their design available for reuse.

- Use of project management methodologies, good coding practices, detailed documentation and version control utilities.

\section{Conclusions and future work}

While this is a work in progress, it has reached a stage in which one can start designing applications and a complete set of experiments on the development board. Most probably a second round of PCBs needs to be designed in order to correct some of the undocumented features (bugs) in both hardware and software. Probably a more modular set of boards will be designed that will be able to hold a major subsystem at a time. This could be a better tradeoff in terms of student's experience and for easy to assemble, bring up and debug. While this platform does not bring up the latest and greatest in terms of technology, its design has been possible through donations from ARM, Altera and Texas Instruments which have been always supportive on creating new and rich learning experiences for students.

Till 20 or 30 years ago some of students were learning and designing systems in which most of the components were discrete and a lot more insight was possible since the hardware and software component were at their reach. This new generation of millennial students work with systems thousands of times more complex to what 20-30 years back student used to, but sometimes is good to go to the basics and let them see what are the systems made of, and that has been lost by integration, miniaturization, reusability, object oriented paradigms to list a few.

The work on this platform will continue and hope that it will be included at several stages in the engineering technology program to try to bring a little more insight on how embedded systems work and their basic components. 


\section{References}

[1] W. Hohl, ARM Assembly Language: Fundamentals and Techniques: CRC, 2009.

[2] S. Furber, "ARM System on a chip Architecture," 2000.

[3] J. O. Hamblen, T. S. Hall, and M. D. Furman, Rapid Prototyping of Digital Systems: SOPC Edition: Springer, 2007. 